\title{
A Probabilistic Model for Dependency Parsing Considering Ascending Dependencies
}

KWANG J. SEO, KI C. NAM, and KEY S. CHOI

Korea Advanced Institute of Science and Technology, Korea

\begin{abstract}
In this study, we propose a new probability model for disambiguation in dependency parsing. In order to enhance accuracy, early dependency models used two paremeters: distance and the part of speech tag of the left sister derived from the word order within a sentence. However, these parameters are not appropriate in free order or elliptical languages such as German, Korean, and Russian. In the proposed model, instead of adopting parameters based on word order, ascending dependency was used to improve accuracy. Ascending dependency is defined as a relationship between a word and any ascendant word in the dependency hierarchy. This approach is useful for free order and the frequently elliptical languages, because in the dependency gremmar the relationship between words is critical. The performance of the three models, including the proposed models of Collins and Eisner, was compared, when they were applied to Korean. These models were trained by 9,000 sentences and tested by 300 sentences sampled from the KIBS tree bank. The result showed that the proposed model works better in accuracy than Collins' model by $2.4 \%$ and Eisner's model by $2.1 \%$.
\end{abstract}

\section{Introduction}

To find the most probable parses, many researchers have tried to extend their grammar formalisms and algorithms to the probabilistic models (Kupiec, 1991; Wright et al., 1991; Lafferty et al, 1992; Schabes, 1992; Briscoe and Carrol, 1993; Han and Choi, 1994). Recently, Collins (1996) and Eisner (1996) tried to extend the formalism by probabilistic models for dependency parsing.

In the dependency grammar, the structure of a sentence is analysed by associating individual words by the labelled dependency links (Mel'cuk, 1988; Covington, 1990). A sentence can be analysed into the syntactic dependencies between individual words as shown in Fig. 1.

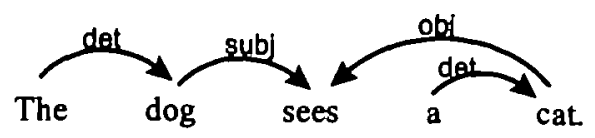

Fig. 1 A dependency parse.

Collins (1996) proposed a probabilistic model to calculate the conditional probability of a dependence parse $\delta$, given a sentence $\omega_{1, n}$ and its POS tag list $t_{1, n}$. Ignoring the concept of baseNP, ${ }^{2}$ this model can be described as in Equation 1.1, where $h_{t}$ is the index of the head word of $\omega_{l}, \Delta_{t}$ is the distance between $\omega_{t}$ and $\omega_{h_{l}}$,

Correspondence: Kwang J. Seo, Department of Computer Science, Korea Advanced Institute of Science and Technology, 373-1, Kusung-dong Yusong-gu, Taejon, 305-701, Korea. E-mail: kjseo@world.kaist.ac.kr

Literary and Linguistic Computing, Vol. 13, No. 2, 1998 and $r_{t}$ is the type of the link (or relationship) between $\omega_{t}$ and $\omega_{h_{i}}$.

$$
\operatorname{Pr}\left(\delta \mid \omega_{1, n}, t_{1, n}\right) \stackrel{\text { def }}{=} \prod_{t=1}^{n} \operatorname{Pr}\left(r_{l} \mid\left\langle\omega_{l}, t_{l}\right\rangle,\left\langle\omega_{h_{l}}, t_{h_{l}}\right\rangle, \Delta_{l}\right)
$$

Also, Eisner (1996) proposed three probabilistic models for a bare-bone dependency parse (BDP), which is a dependency parse without considering the types of dependencies as in Fig. 2.

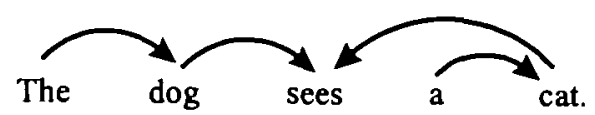

Fig. 2 A bare-bone dependency parse

The recursive generation model, which is the simplest and best dependency parsing model among the three proposed models by Eisner (1996), can be written as in Equation 1.2, where $s_{t}$ is the index of the closest left sister word.

$$
\operatorname{Pr}\left(\delta, \omega_{1, n}, t_{1, n}\right)=\prod_{i=1}^{\text {def }} \operatorname{Pr}\left(<\omega_{l}, t_{l}>\mid t_{s_{l}},<\omega_{h_{i}}, t_{h_{i}}>\right)
$$

These early models for dependency parsing used identifiers of dependent and head words. In addition, they consider parameters, distance, and POS tags of the left sister derived from the word order within a sentence in order to improve accuracy. However, these parameters based on the word order are not suitable in a non-Western language syntax such as Korean, in which the order of words is relatively free within a sentence and frequently dependent words are omitted. In the free order and frequently elliptical languages, the distance between the related two words and the tag of the left sister word varies too much. Furthermore, the frequencies of these parameter values may not have any consistent patterns and so they may not be meaningful in disambiguation.

In this paper, a new model for a BDP is proposed. For higher accuracy in performance, the proposed model considers ascending dependencies in the dependency hierarchy, instead of using parameters based on the word order within a sentence.

\section{The Proposed Probabilistic Model Based on Ascending Dependency}

\subsection{Ascendants and formalism of the $B D P$}

The BDP is a rooted tree having the sentence head as the root. In the rooted tree, every node except the root has only one outgoing arrow and there are no cycles.

Let us assume that $X$ and $Y$ are nodes and there is a 
directed path having length $r$ from $X$ to $Y$ in the rooted tree. In this case, $\mathrm{Y}$ is the ascendant of $\mathrm{X}$ whose order is $r$ and is called the rorder ascendant. For the proposed model, $\boldsymbol{k}$-ascendants $(\boldsymbol{k}$-AS) of a word are defined as a list consisting of ascendants whose orders are less than $k$. Thus, 1-AS of a node consists of only a 1-order ascendant, 2-AS consists of 1- and 2-order ascendants, and $\infty$-AS consists of all ascendants to the root. For example, in Fig. 2, the 1-order ascendant of 'the' is 'dog', and the 2-order ascendant is 'sees'. Thus, 2-AS of 'the' is ['dog', 'sees'].

In our study, BDP is formalized by using $k$-AS to describe statistical language modelling. The BDP has two kinds of information: the dependency relationship between words and the word order in a sentence. $A$ binary relationship can be represented by an ordered pair and an order can be represented by a list. Applying these structures, the BDP can be represented by the list of ordered pairs involving a dependent and its head $\left[\left(\omega_{l}, h_{t}\right) \mid i=1, n\right]$. Also, the list of ordered pairs can be represented by an ordered pair of two lists; the first is the list of dependents and the second is the list of head $\left(\left[\omega_{i} \mid i\right.\right.$ $\left.=1, n],\left[h_{t} \mid i=1, n\right]\right)$. Let the head of the sentence head be ' $\$$ '. Figure 2 is represented as in Equations 2.1 and 2.2.

[(The, dog), (dog, sees), (sees, \$), (a, cat.), (cat., sees)]

([The, dog, sees, a, cat.], [dog, sees, \$, cat., sees])

In the ordered pair of two lists, the list of the dependents is another representation of a sentence and the list of the head is identical with the list of 1-AS. Therefore, the BDP can be represented uniquely by a pair of a sentence and a list of 1-AS as shown in Equation 2.3. Because 1-AS is a sublist of 2-AS, the BDP can be expressed uniquely by a pair of sentences and a list of 2-AS as shown in Equation 2.4.

([The, dog, sees, a, cat.], [[dog], [sees], [, [cat.], [sees]])

([The, dog, sees, a, cat.], [[dog, sees], [sees], [], [cat., sees], [sees]])

Generalizing these representations, the BDP can be represented uniquely by a sentence and a list of $\boldsymbol{k}$-AS for $k \geq 1$.

\subsection{A Probability Model Using Ascending Dependencies}

From the viewpoint of probability, as shown in Equation 2.5, a disambiguating parsing is a procedure $\Phi$, to find a parse $\delta$ that maximizes the probability of it, given a sentence $\omega_{1, n}$ (Merialdo, 1994). In this section, we introduce a new model of the joint probability of a $\mathrm{BDP}$ and a sentence.

$$
\begin{aligned}
\Phi\left(\omega_{1, n}\right) & =\underset{\sigma}{\operatorname{argmax}} \operatorname{Pr}\left(\delta \mid \omega_{1, n}\right) \\
& =\arg \max _{\delta} \operatorname{Pr}\left(\delta, \omega_{1, n}\right)
\end{aligned}
$$

BDP $\delta$ can be replaced by a pair composed of a sentence and a $k$-AS list $H_{1, n}^{k}$. Thus, the probability can be rewritten by the probability of $k$-AS list $H_{1, n}^{k}$ for the given sentence $\omega_{1, n}$ as shown in Equation 2.6.

$$
\begin{aligned}
\operatorname{Pr}\left(\delta, \omega_{1, n}\right) & =\operatorname{Pr}\left(\omega_{1, n}, H_{1, n}^{k}, \omega_{1, n}\right) \\
& =\operatorname{Pr}\left(H_{1, n}^{k}, \omega_{1, n}\right)
\end{aligned}
$$

To develop the joint probability (Equation 2.6), this paper uses two formulae. The first formula (Equation 2.7 ) is for the conditional probability of $k$-AS.

$\operatorname{Pr}\left(H_{t}^{k} \mid \omega_{j}, H_{j}^{k}\right.$ others $)=1$, when $\omega_{j}$ is the head of $\omega_{t}$

Let the r-order ascendant of $\omega_{l}$ be $h_{l}^{r}$, then $H_{t}^{k}$ is $h_{1}^{1, k}$. Because $h_{i}^{1}$ is $\omega_{l}$ and $h_{i}^{r}$ is $h_{j}^{r-1}, H_{t}^{k}$ can be expressed as $\left[\omega_{l}\right.$, $\left.h_{j}^{1, k-1}\right]$ or $\left[\omega_{j}, H_{j}^{k-1}\right]$. Thus, Equation 2.7 is always true.

The second formula (Equation 2.8) is for the conditional probability of a word. This formula is the independence assumption that the probability of $\omega_{t}$ is dependent only on the $k$-AS.

$$
\operatorname{Pr}\left(\omega_{\imath} \mid H_{l}^{k}, \text { others }\right) \approx \operatorname{Pr}\left(\omega_{\imath} \mid H_{l}^{k}\right)
$$

From the linguistic viewpoint, the dependents of a word are syntactic components to fulfil their meanings. That is to say, a word in a sentence exists only due to the requirement of its head (1-ordered ascendant). In turn, the head exists due to its own head (2-ordered ascendant); the 2-ordered ascendant has an effect indirectly on the existence of the word. Therefore, all ascendants influence the existence of the dependent word recursively. Ascending dependencies are the relationships of these indirect influences (see Fig. 3).

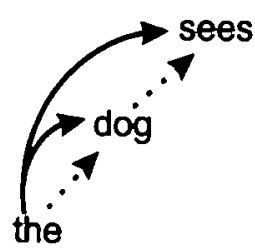

Fig. 3 Ascending dependencies (notated by solid line) of 'the' in Fig. 2.

In this thesis, the independence assumption (Equation 2.8) is based on the concept of ascending dependencies, and the order of influencing ascendant is limited up to $k$ in order to avoid computational complexity and to be efficient in performance.

Now, let us derive our model using the above formulae. In order to see how the conditional probability (Equation 2.6) is derived, take a look at the small BDP in Fig. 2 having 2-AS. The probability of the BDP is shown as the joint distribution probability of Equation 2.10 , when the random variables are assigned as shown in Equation 2.9.

$\omega_{1}=$ 'The', $\omega_{2}=$ 'dog', $\omega_{3}=$ 'sees', $\omega_{4}=$ 'a', $\omega_{5}=$ 'cat'

$H_{1}^{2}=\left[\omega_{2}, \omega_{3}\right], H_{2}^{2}=\left[\omega_{3}\right], H_{3}^{2}=[], H_{4}^{2}=\left[\omega_{5}, \omega_{3}\right], H_{\xi}=\left[\omega_{3}\right]$

Equation 2.10 is rewritten into Equation 2.11 by reordering the variables from the top downwards in the dependency hierarchy. Thus, Equation 2.11 is transformed into Equation 2.12 by applying the chain rule.

$$
\begin{aligned}
& \operatorname{Pr}\left(H_{1}^{2}, H_{2}^{2}, H_{3}, H_{4}^{2}, H_{\xi}, \omega_{1}, \omega_{2}, \omega_{3}, \omega_{4}, \omega_{5}\right) \\
& =\operatorname{Pr}\left(H_{3}^{2}, \omega_{3}, H_{2}^{2}, \omega_{2}, H_{5}^{2}, \omega_{5}, H_{1}^{2}, \omega_{1}, H_{4}^{2}, \omega_{4}\right) \\
& =\operatorname{Pr}\left(H_{3}^{2}\right) \\
& \times \operatorname{Pr}\left(\omega_{3} \mid H_{3}^{2}\right) \\
& \times \operatorname{Pr}\left(H_{2}^{2} \mid \omega_{3}, H_{3}^{2}\right) \\
& \times \operatorname{Pr}\left(\omega_{2} \mid H_{2}^{2}, \omega_{3}, H_{3}^{2}\right) \\
& \times \operatorname{Pr}\left(H_{\xi}^{2} \mid \omega_{2}, H_{2}^{2}, \omega_{3}, H_{3}^{2}\right) \\
& \times \operatorname{Pr}\left(\omega_{5} \mid H_{\xi}^{2}, \omega_{2}, H_{2}^{2}, \omega_{3}, H_{3}^{2}\right)
\end{aligned}
$$

Literary and Linguistic Computing, Vol. 13, No. 2, 1998 


$$
\begin{aligned}
& \times \operatorname{Pr}\left(H_{1}^{2} \mid \omega_{5}, H_{3}, \omega_{2}, H_{2}^{2}, \omega_{3}, H_{3}^{2}\right) \\
& \times \operatorname{Pr}\left(\omega_{1} \mid H_{1}^{2}, \omega_{5}, H_{3}^{2}, \omega_{2}, H_{2}^{2}, \omega_{3}, H_{3}^{2}\right) \\
& \times \operatorname{Pr}\left(H_{4}^{2} \mid \omega_{1}, H_{1}^{2}, \omega_{5}, H_{3}^{2}, \omega_{2}, H_{2}^{2}, \omega_{3}, H_{3}^{2}\right) \\
& \times \operatorname{Pr}\left(\omega_{4} \mid H_{4}^{2}, \omega_{1}, H_{1}^{2}, \omega_{5}, H_{3}, \omega_{2}, H_{2}^{2}, \omega_{3}, H_{3}^{2}\right)
\end{aligned}
$$

In Equation 2.12, by applying Equation 2.7, the third term, $\operatorname{Pr}\left(H_{2} \mid \omega_{3}, H_{3}\right)$, becomes 1 , because the head of $\omega_{2}\left(\omega_{3}\right)$ and the head's $k$-AS $\left(H_{3}\right)$ exist in the condition. Likewise, the fifth, the seventh, and the ninth term become 1 . Also, by applying the independence assumption (Equation 2.8), the fourth, the sixth, the eighth, and the tenth term can be replaced by the 2-gram conditional probabilities, each involving a word and its $k$ AS. Finally, Equation 2.10 can be represented by the probability of Equation 2.13.

$\operatorname{Pr}\left(H_{3}^{2}\right) \times \operatorname{Pr}\left(\omega_{3} \mid H_{3}^{2}\right) \times \operatorname{Pr}\left(\omega_{2} \mid H_{2}^{2}\right) \times \operatorname{Pr}\left(\omega_{5} \mid H_{5}^{2}\right) \times$

$\operatorname{Pr}\left(\omega_{1} \mid H_{1}^{2}\right) \times \operatorname{Pr}\left(\omega_{4} \mid H_{4}^{2}\right)$

$=\operatorname{Pr}\left(H_{3}\right) \times \prod_{t=1}^{5} \operatorname{Pr}\left(\omega_{t} \mid H_{t}^{2}\right)$

Let $s$ be the index of the sentence head word, then Equation 2.13 is generalized into Equation 2.14. In Equation 2.14, $\operatorname{Pr}\left(H_{s}^{k}\right)$ can be removed, because $H_{s}^{k}$ is always a null list []. Then Equation 2.14 is reduced into Equation 2.15 which is our model.

$$
\begin{aligned}
\operatorname{Pr}\left(\delta, \omega_{1, n}\right) & =\operatorname{Pr}\left(H_{s}^{k}\right) \times \prod_{l \square 1}^{n} \operatorname{Pr}\left(\omega_{\imath} \mid H_{l}^{k}\right) \\
& =\prod_{l=1}^{n} \operatorname{Pr}\left(\omega_{l} \mid H_{l}^{k}\right)
\end{aligned}
$$

\subsection{Training the Model}

This section describes a method for estimation of the conditional probability, $\operatorname{Pr}\left(\omega_{\imath} \mid H_{l}^{k}\right)$. If we have some available syntactic corpus, we can estimate it by computing the relative frequencies. Let $\mathrm{C}\left(\omega_{t}, H_{l}^{k}\right)$ be the number of times that a given word $\omega_{t}$ appears with $H_{t}^{k}$ and let $\mathrm{C}\left(H_{t}^{k}\right)$ be the number of times that a given $H_{t}^{k}$ appears in this corpus. Then, the conditional probability can be estimated by the relative frequency, $\mathrm{E}\left(\omega_{\imath}, H_{l}^{k}\right)$, as shown in Equation 2.16.

$$
\operatorname{Pr}\left(\omega_{\imath} \mid H_{\imath}^{k}\right) \cong \mathrm{E}\left(\omega_{\imath}, H_{i}^{k}\right)=\frac{\mathrm{C}\left(\omega_{\imath}, H_{\imath}^{k}\right)}{\mathrm{C}\left(H_{\imath}^{k}\right)}
$$

This estimation may have the sparse data problem which confronted other statistical models. To avoid this data sparseness, we interpolate this relative frequency with Equation 2.17.

$$
\begin{aligned}
& \mathrm{E}\left(\omega_{\imath}, H_{t}^{k}\right)=\lambda_{k} \mathrm{E}\left(\omega_{\imath}, H_{l}^{k}\right)+\lambda_{k-1} \mathrm{E}\left(\omega_{\imath}, H_{\imath}^{k-1}\right) \\
& +\ldots+\lambda_{1} \mathrm{E}\left(\omega_{\imath}, H_{\imath}^{1}\right)+\lambda_{0} \mathrm{E}\left(\omega_{\imath}, H_{\imath}^{0}\right)
\end{aligned}
$$

The coefficient $\lambda$ is computed by using a simple method as shown in Equation 2.18, when $\lambda_{k+1}$ is 0 . As a variation of the method of Jelinek and Mercer (1980), this method has a desirable property; i.e. as the training corpus increases, the coefficient $\lambda$ for the more specific event tends to increase.

$$
\lambda_{r}=\left\{\begin{array}{c}
\left(1-\sum_{p=r+1}^{k} \lambda_{p}\right) \cdot \frac{\mathrm{C}\left(H_{l}^{r}\right)}{\mathrm{C}\left(H_{i}^{r}\right)+1}, r \geq 1 \\
1-\sum_{p=1}^{k} \lambda_{p}, r=0
\end{array}\right.
$$

\section{Application to Korean BDP}

Korean sentences are composed of word phrases delimited by spaces. For Korean parse, a word phrase is usually used as a syntactic unit. For our research, Korean BDP consisting of word phrases is used as the target of analysis.

A word phrase can be divided into two parts; one is a content word and the other is a function word. In our study, the relationship between two word phrases is determined by the content word in the head phrase and the functional word in the dependent phrase.

For example, Table 1 presents the content and function word elements of the word phrases in the sentence given in 3.1. The content word 'ka' of 'ka-nda', which is the head phrase, has a sub-categorization of a direction or a place. Also, the functional word ' $\theta$ ' in 'hakkyo- $\theta$ ', which is the dependent phrase of ' $k a-n d a$ ', represents a case of a place. Therefore, the relationship between two word phrases 'hakkyo- $\theta$ ' and 'ka-nda' is determined by the content word ' $k a$ ' in the head phrase and the functional word ' $\theta$ ' in the dependent phrase.

'na-nun hakkyo-e ka-nda.'

(which, in English, means, 'I am going to school')

Table 1 The word phrases in 3.1

\begin{tabular}{lllllll}
\hline Word phrase & \multicolumn{2}{l}{ 'na-nun' } & \multicolumn{2}{l}{ 'hakkyo-e' } & \multicolumn{2}{c}{ 'ka-nda' } \\
& CONT & FUNCT & CONT & FUNCT & CONT & FUNCT \\
\hline Word & 'na' & 'nun' & 'hakkyo' & 'e' & 'ka' & 'nda' \\
In English & I & [subject] & School & To & Go & be-ing \\
\hline
\end{tabular}

Considering the above characteristics of Korean word phrases, the dependent word parameter $\omega_{1}$ in the independence assumption (Equation 2.8) is replaced by its functional word $f_{i}$. Also, the list of the ascendants, $H_{i}^{k}$, is replaced by the list of the content words notated by $\mathrm{CH}_{\imath}^{k}$.

$$
\operatorname{Pr}\left(\omega_{\imath} \mid H_{\imath}^{k} \text {, others }\right) \approx \operatorname{Pr}\left(f_{l} \mid C H_{\imath}^{k}\right)
$$

When applying the assumption (Equation 3.2) to Korean BDP, the model for Korean BDP is expressed as in Equation 3.3.

$$
\operatorname{Pr}\left(\omega_{1, n}, \delta\right) \stackrel{\text { def }}{=} \prod_{\imath=1}^{n} \operatorname{Pr}\left(f_{l} \mid C H_{\imath}^{k}\right)
$$

\section{Experiments}

The goal of our study is to show that the proposed model works more efficiently than the previous models (e.g. Collins, 1996; Eisner, 1996), when applied to nonWestern languages having the relatively free order of words within a sentence and frequently omitting the dependent words.

\subsection{Training Data and Measures}

The three models were trained by 9,000 sentences and tested on 300 sentences of the KIBS tree bank ${ }^{2}$ which is on average 10.5 word phrases in length. In order to evaluate the performance change as training proceeds, the models were trained by nine phases in which each phrase consists of 1,000 sentences. 
The performance of the models was evaluated by the number of rules automatically extracted from training data and the precision, which is the percentage of words correctly attached to their parents. The number of rules extracted from training data reflects the degree of the computing complexity of the models. The more complex a computation is, the more rules are generated and the more sharply the rates of the number of the rules increases. Thus, when a model works at the same accuracy with fewer rules, it means that the model performs better than the other models.

\subsection{Comparison of the Proposed Model with those of Collins (1996) and Eisner (1996)}

The performance of the three models was compared by applying them to the Korean BDP consisting of word phrases. To avoid data sparseness, the lexical information in a word was not used. Only POS categories are used.

In order to compare the performance of the three models fairly, a 3-gram model (i.e. a 2-AS model) was used in the proposed model as in the other two models, ${ }^{3}$ and the other two models were modified to deal with the characteristic of Korean phrases. Equations 4.1, 4.2, and 4.3 present the three modified models for comparison, where $c_{l}$ is the content word of the word phrase $\omega_{t}$, $h_{l}$ is the index of the 1-order ascendant of $\omega_{l}$, and $g_{l}$ is the index of the 2-order ascendant of $\omega_{l}$. In Equation 4.2 model, the link type $r_{t}$ is restricted only to one type related to the BDP.

$$
\begin{aligned}
& \text { 2-AS model } \prod_{i=1}^{n} \operatorname{Pr}\left(\operatorname{POS}\left(f_{\imath}\right) \mid \operatorname{POS}\left(c_{h_{l}}\right), \operatorname{POS}\left(c_{g_{l}}\right)\right) \\
& \text { Collins model } \prod_{t=1}^{n} \operatorname{Pr}\left(r_{t}\left|\operatorname{POS}\left(f_{t}\right)\right| \operatorname{POS}\left(c_{h_{l}}\right), \Delta_{l}\right) \\
& \text { Eisner model } \prod_{i=1}^{n} \operatorname{Pr}\left(\operatorname{POS}\left(f_{i}\right) \mid \operatorname{POS}\left(f_{s_{l}}\right), \operatorname{POS}\left(c_{h_{l}}\right)\right)
\end{aligned}
$$

Figure 4 shows the number of rules each model extracts as the number of training sentences increases. While the number of rules increases sharply in the Eisner model as training sentences are added, in the proposed model and in that of Collins, the number of rules increases gradually. Comparing the proposed model with the Collins model in terms of computational complexity, the proposed model extracts more rules than the Collins model. Figure 4 indicates that in respect of the models' complexity, the Eisner model is the most complex among the three and the Collins model is the simplest.

Figure 5 shows the precision change as the number of training sentences increases. The figure indicates that the proposed model performs accurately in all training

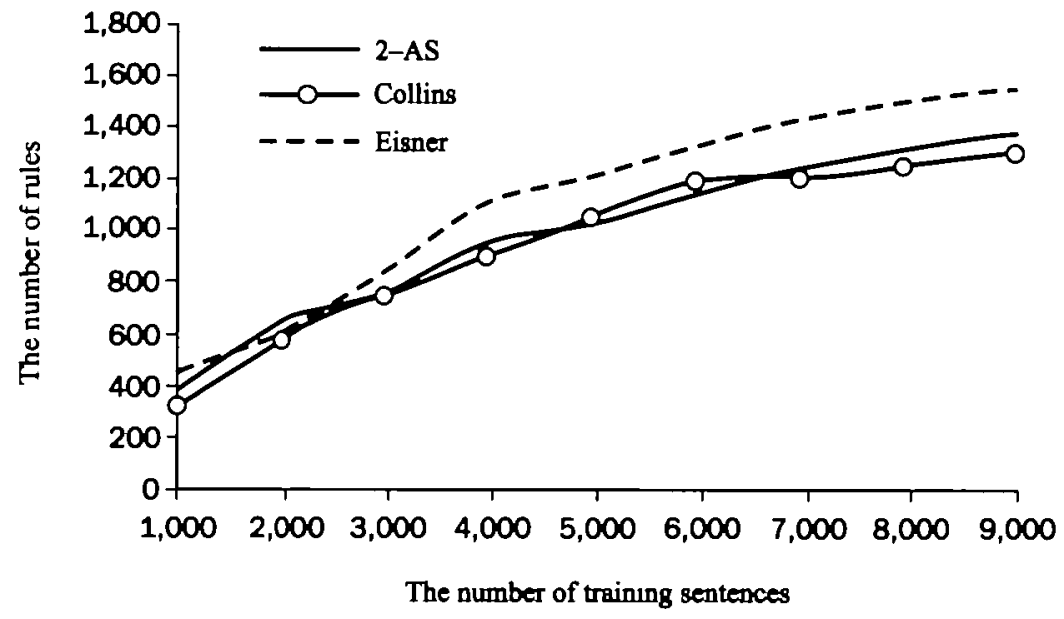

Fig. 4 The number of rules as the training sentences are accumulated

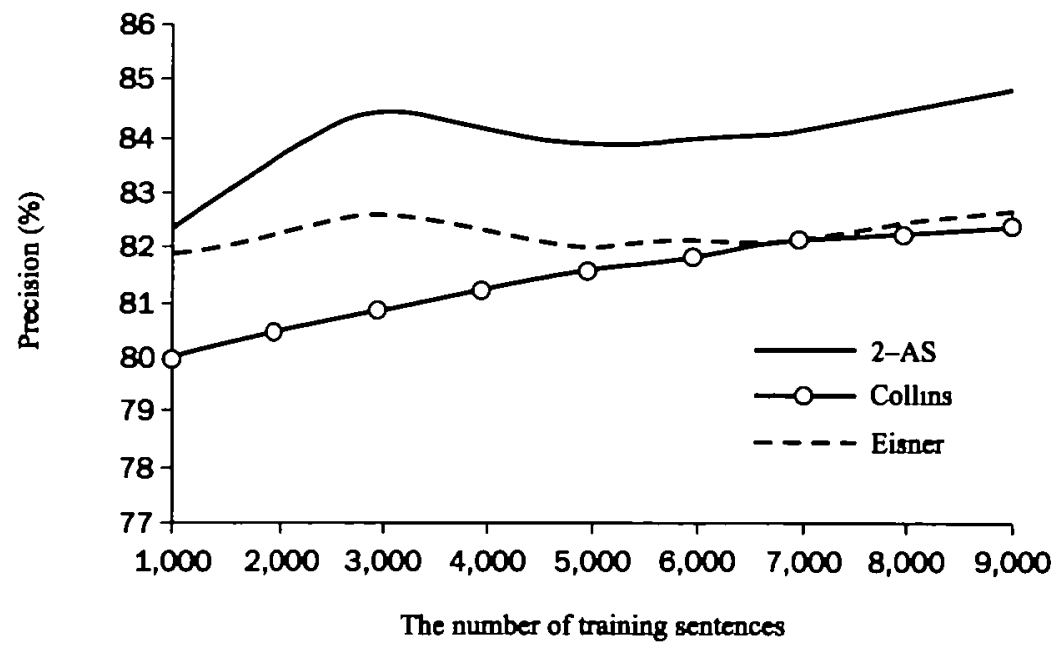

Fig. 5 The accuracy change as the traning sentences are accumulated 
phases. After being trained on 9,000 sentences, the accuracy of the present model was $84.8 \%$, whereas the accuracy of the Eisner and Collins models was 82.7 and $82.4 \%$ respectively.

In summary, in terms of complexity, the Eisner model is the most complex and the Collins model is the least complex. Regarding performance accuracy, the proposed model works most accurately and the Collins model performs worst. In considering the number of rules extracted from the training sentences and the performance accuracy, the proposed model is the most suitable for Korean. The difference in performance between the proposed model and the other two models occurs due to the characteristics of Korean. Whereas the other two models are based on fixed word order and thus cannot reflect the flexible phrase order within a sentence in Korean, the proposed model does not have a basis in fixed word order but in ascending dependency, and this ascending dependency solves the problems occurring due to the free order of the phrases and the frequent ellipsis.

\section{Summary}

We propose here a new probability model for dependency parsing using ascending dependency in the dependency hierarchy.

Early models of dependency parsing (e.g. Collins, 1996; Eisner, 1996) used contexts based on the word order, such as the distance and POS tags of the left sister, in order to enhance accuracy. However, considering these parameters in free order or elliptical languages may not be appropriate, because these parameters vary frequently depending on the word order.

In the proposed model, the ascending dependency in the dependency hierarchy is used, instead of the parameters based on word order. The ascending dependency is the relationship between a word and its ascending head. Considering the higher heads as well as the immediate head reflects the influence of the higher dependencies as well as the immediate dependency, while preserving the merit of the dependency grammar in which individual words need not be restricted to a particular word order unlike a constituency structure.

The basic probability in the proposed model can be estimated by the relative frequency. This estimation is likely to face the data sparseness problem as do other statistical models. To avoid this data sparseness, the proposed model uses an interpolating method which is a variation of that of Jelinek and Mercer (1980).

The performance of the three models [i.e. the proposed model, Collins (1996), and Eisner (1996)] was compared for Korean BDP. For fair comparison, the proposed model used 3-grams and the other two models were modified to deal with the characteristics of Korean word phrases.

The results of the experiment using 9,300 sentences from the KIBS tree bank showed that the proposed model performs better in terms of accuracy than the Collins and Eisner models by 2.4 and by $2.1 \%$ respectively.

\section{Notes}

1. The KIBS tree bank consists of $\sim 30,000$ sentences. each of which is on average 11.4 word phrases in length. KIBS (Korea Information Base System) is a part of a contract by the Center for Korean Language Engineering funded by the Ministry of Science and Technology and the Ministry of Education and Athletics.

2. A baseNP is a non-recursive NP, i.e. none of tts child constituents are NPs.

3. In the Collins model (Equation 1.1), the link type is restricted only to one type considering BDPs. Thus, the Collins model is also regarded as a 3-gram model.

\section{References}

Briscoe, T. and Carrol, J. (1993). Generalized Probabulistuc LR Parsing of Natural Language (Corpora) with Unification-based Grammars. Computational Lingusstics, 19: 25-57.

Collins, M. (1996). A New Statistical Parser Based on Bigram Lexical Dependencies. In Proceedings of the 34th Annual Meeting of the Association for Computational Linguistics. Santa Cruz, Calıfornıa, pp. 184-91.

Covington, M. (1990). A Dependency Parser for Vanableword-order Languages. Research Report AI-1990-02, University of Georgia.

Eisner, J. (1996). Three New Probabilistic Models for Dependency Parsing: An Exploration. In Proceedings of 16th International Conference on Computational Lingusstics. Copenhagen, pp. 340-5.

Han, Y.S. and Chol, K.S. (1994). A Reestimation Algorithm for Probabilistic Recursive Transition Network. In Proceedings of the 15th International Conference on Computational Linguistics. Kyoto, Japan, pp. 859-64.

Jelinek, F. and Mercer, R.L. (1980). Interpolated Estimation of Markov Source Parameters from Sparse Data. In Proceedings of Workshop on Pattern Recognition in Practice, pp. 381-97.

Kupiec, J. (1991). A Trellis-based Algorithm for Estimating the Parameters of Hidden Stochastic Context-free Grammar. In Proceedings of Speech and Natural Language Workshop. Pacific Grove, pp. 241-6.

Lafferty, J., Sleator, D., and Temperley, D. (1992). Grammatical Trigrams: A Probabilistic Model of Link Grammar. In Proceedings of AAAI Fall Symposium Series: Probabllstic Approaches to Natural Language. Cambridge, pp. 89-97.

Mel'cuk, I.A. (1988). Dependency Syntax: Theory and Practice. State University of New York Press.

Merialdo, B. (1994). Tagging English Text with a Probabilistic Model. Computational Linguzstics, 20: 155-71.

Schabes, Y. (1992). Stochastic Lexicalized Tree-adjoining Grammars. In Proceedings of the 14th International Conference on Computational Linguistics. Nantes, pp. 426-32.

Wright, J., Wrighley, E., and Sharman, R. (1991). Adaptive Probabilistıc Generalized LR Parsing. In Proceedings of the 2nd Internatonal Workshop on Parsing Technologies. Cancun, Mexico, pp. 154-68. 
